\title{
Atypical Presentation of a Sinonasal Adenocarcinoma Clinically Mimicking a Mucocele: Case Report
}

\author{
Radhi M El Hafed", Ahmadou A, Chebaatha A, Oukessou Y, Rouadi S, Abada RA, Roubal \\ $M$ and Mahtar $M$
}

Department of ENT-HNS, Casablanca, Morocco

\begin{abstract}
Sinonasal adenocarcinomas are rare, it represent 4 to $20 \%$ of nasosinusian carcinomas, the latest WHO 2017 classification distinguishes two types: Intestinal type adenocarcinomas (ITAC) and non-intestinal type, with different prognosis; intestinal adenocarcinomas being more aggressive with a mortality rate around 53\%; nasal obstruction, epistaxis, and rhinorrhea are common symptoms, the rhinoscopy found multiple fleshy, bleeding formations in contact, occupying nasal cavities first evokes a mucocele.
\end{abstract}

We present the case of a 53-year-old patient who reported a rapidly growing soft mass on his nasal pyramid for 10 months, with nasal obstruction, bilateral exophthalmos and blindness of the right eye, on the CT scan of sinuses, we observed a heterogeneous formation, associated with adjacent bone destruction with intracranial extension, directing the diagnosis to a neoplasm sinonasal malignant; A deep biopsy under general anesthesia was made, the histological study concluded to intestinal sinonasal adenocarcinoma; which are rare.

High grade sinonasal adenocarcinomas are locally aggressive tumors often diagnosed at an advanced stage. Their treatment is mainly surgical and the prognosis depends essentially on the clinical stage; therefore the diagnosis should not be delayed.

Our case underlines the importance to always maintain a high clinical index of suspicion of malignancy, in particular in cases of extensive mucocele

Keywords

Adenocarcinoma, Nasal Cavity, Mucocele

\section{Introduction}

Carcinomas of the nasal cavity and paranasal sinus are rare, with an annual incidence of less than 1 per 100,000 individuals [1,2]. The latest WHO 2017 classification distinguishes two types of adenocarcinomas: Intestinal type adenocarcinomas (ITAC) and non-intestinal type, with different prognosis implications [3]; intestinal type adenocarcinomas are more aggressive with a mortality rate of around 53\% [4], Sinonasal intestinal type adenocarcinomas are clinically aggressive and diagnosed most often at an advanced stage with a mortality of $53 \%$ [4]; however metastases are relatively rare, and the prognosis is mainly attributed to clinical stage and recurrences frequency [5]. Sinonasal intestinal type adenocarcinomas makes up only a small percent of all nasal and paranasal sinus malignancies [3], they occur sporadically or are associated with occupational exposure to hardwood and leather dust, nickel, and possibly smoking [6]. The relationship between exposure to wood dust and the development of intestinal-type adenocarcinoma has been established by numerous epidemiological studies [6-8]. The preferential site of sinonasal adenocarcinoma is the ethmoidal sinus [3] and men are 5 times as likely to develop these tumours as women [4]. Symptoms are often a common rhinologic pathology [9] Radiologic examination is based on computed tomography (CT) scan and magnetic resonance imagery (MRI). The most commonly employed treatment has been a combined treatment associating surgery and radiotherapy. We describe a rare case of sinonasal adenocarcinoma which presents clinical atypical mimicking a mucocele.

*Corresponding author: Radhi M El Hafed, Department of ENTHNS, Casablanca, Morocco

Accepted: January 09, 2021

Published online: January 11, 2021

Citation: El Hafed RM, Ahmadou A, Chebaatha A, et al. (2021) Atypical Presentation of a Sinonasal Adenocarcinoma Clinically Mimicking a Mucocele: Case Report. J Head Neck Surg 3(1):119121

Copyright: (C) $2021 \mathrm{El}$ Hafed RM, et al. This is an open-access article distributed under the terms of the Creative Commons Attribution License, which permits unrestricted use, distribution, and reproduction in any medium, provided the original author and source are credited. 


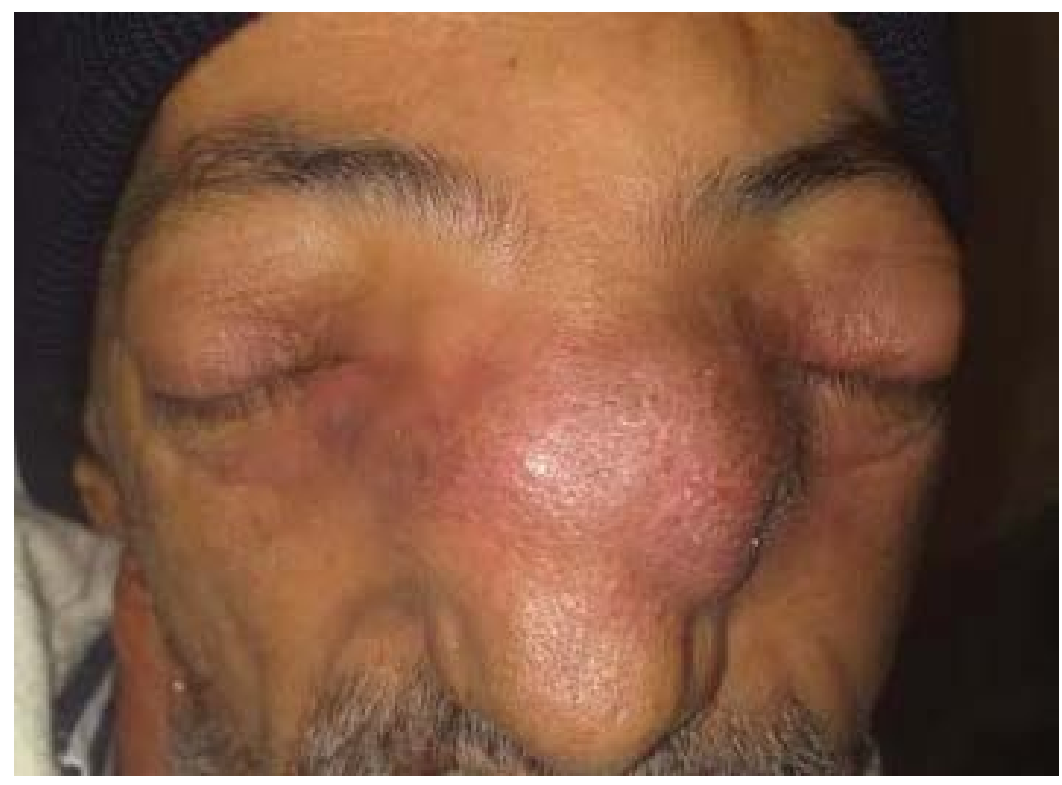

Figure 1: Prefistulization nasal mass with bilateral exophthalmos.

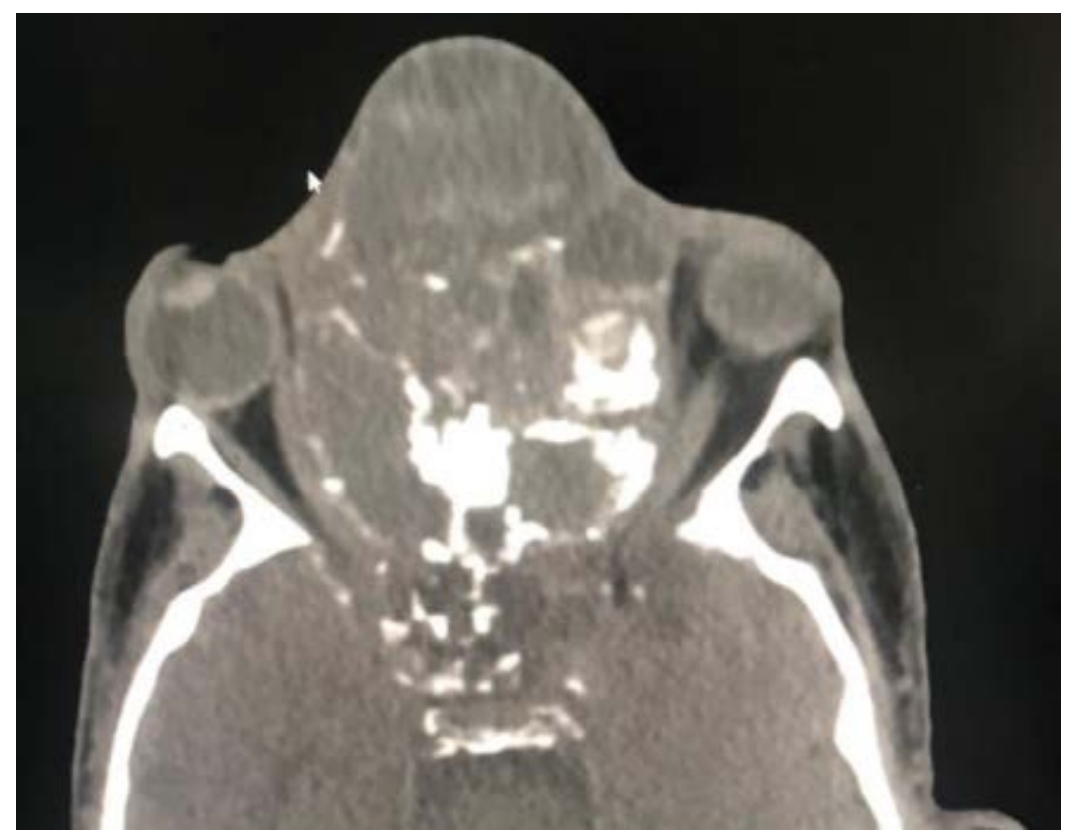

Figure 2: $\mathrm{CT}$ of the paranasal sinuses in the soft tissue window, showing an expansive lesion, with areas of necrosis, bone destruction with bilateral and intracranial orbital dissemination.

\section{Case Presentation}

We report the case of a 53-year-old patient, with 10-years history of smoke; reported a rapidly growing soft mass on his nasal pyramid for 10 months, with bilateral exophthalmos, blindness in the right eye and permanent bilateral nasal obstruction, associated with intermittent epistaxis, and anosmia. (Figure 1). The patient subsequently reported insomnia, and intense headache that was resistant to symptomatic medical treatment (paracetamol). The rhinoscopy found multiple fleshy, bleeding formations in contact, occupying nasal cavities; ophthalmologic examination was made objectifying a right optical atrophy and a beginning on the left one. The rest of the examination was without particularities.

CT shows a heterogeneous, expansive formation with hypodense areas in relation to necrosis and soft tissue areas, occupying the entire sinus and nasal cavity with intracranial extension, associated to adjacent bone remodeling and destruction, reducing both orbital cavities, laterally displacing both optic nerves, predominantly right, causing bilateral exophthalmos (Figure 2). Two biopsies carried out were not specific; a third deep biopsy was performed under general anesthesia, which histological examination concluded to a high grade intestinal type adenocarcinoma.

After announcing to the patient the diagnosis and detail- 
ing the therapeutic modalities as well as the prognosis of his disease, the patient did not adhere and he refused to continue the treatment.

\section{Discussion}

Sinonasal intestinal-type adenocarcinoma is a rare neoplasm, with local aggressively behavior and high local recurrence rates [10]. The literature review showed that malignant neoplasms of the sinonasal tract usually present late; $74.7 \%$ of all patients was classed stage III and IV disease at the diagnosis [11-16]. The clinical presentation of this tumor is not specific and can simulate a benign tumor, by the presence of nasal obstruction, and epistaxis, our patient presents in addition, clinical signs which predict a local extension such as exophthalmos and intense headaches. The patient was a 53-year-old man who had age and sex risks for sinonasal neoplasms, but no history of exposure to environmental carcinogens, such as leather or wood dust. After, rhinoscopy; we was more oriented to mucocele. CT scan with intravenous contrast is essential to localize the tumor and defines eventual extensions to adjacent structures; the skull of base, orbit and the deep spaces of the face. Additionally, MRI with intravenous contrast complements $\mathrm{CT}$ by identifying neoplastic dissemination in the soft tissues. In our case, the massive local extension on the scanner, challenged us on the diagnosis of mucocele, and we had to repeat the biopsy to obtain a diagnosis. In addition; mucoceles are benign cystic tumours, arising at the expense of the paranasal sinus mucosa, lined by non-neoplastic epithelium, and containing usually sterile mucus [17]. Given the key role of histology in guiding treatment, it is important that biopsy is obtained with precision and histologic slides are analyzed by a pathologist with specific expertise [18].

Surgery is the most commonly used treatment modality for SNAC; and obtaining negative surgical oncologic margins is essential for tumor control [5]. The endoscopic surgery is the gold standard for the treatment of sinonasal adenocarcinoma, it conditions the prognosis; and adjuvant radiotherapy is recommended in advanced stages and high grade tumors of malignancy [18], moreover advanced stage at diagnosis is acknowledged to have a strong impact on prognosis and, in particular, on the probability of recurrence [19].

Concerning prognosis, disease-related death of sinonasal adenocarcinoma is typically attributed to local recurrence, which occurs in approximately half of cases $[17,20]$. Metastases are relatively uncommon in sinonasal adenocarcinomas and are usually quoted as occurring in $20 \%$ of cases. Lymph node spread occurs in roughly $10 \%$ of cases. This behavior underlies the treatment objective of preventing locoregional recurrence and the importance of early diagnosis to manage this neoplasm.

Our case underlines the importance to always maintain a high clinical index of suspicion of malignancy, in particular in cases of extensive mucocele, in order not to delay the diagnosis

\section{Conflict of Interest}

No conflict of interest.

\section{References}

1. Jin Y, Mertens F, Arheden K, et al. (1995) Karyotypic features of malignant tumors of the nasal cavity and paranasal sinuses. Int J Cancer 60: 637-641.

2. Khademi B, Moradi A, Hoseini S, et al. (2009) Malignant neoplasms of the sinonasal tract: Report of 71 patients and literature review and analysis. Oral Maxillofac Surg 13: 191-199.

3. El-Naggar AK,Chan JKC, Grandis JR, et al. (2017) WHO Classification of Head and Neck tumours. ( $4^{\text {th }}$ Edn), International Agency for Research on Cancer.

4. Kennedy MT, RCK Jordan, KW Berean, et al. (2004) Expression pattern of CK7, CK20, CDX-2, and villin in intestinal-type sinonasal adenocarcinoma. J Clin Pathol 57: 932-937.

5. Shay A, Ganti A, Raman A, et al. (2020) Survival in low-grade and high-grade sinonasal adenocarcinoma: A national cancer database analysis. The Laryngoscope 130: E1-E10.

6. Barnes L (1986) Intestinal-type adenocarcinoma of the nasal cavity and paranasal sinuses. Am J Surg Pathol 10: 192-202.

7. Mensi C, Consonni D, Sieno C, et al. (2013) Sinonasal cancer and occupational exposure in a population-based registry. Int J Otolaryngol 2013: 1-7.

8. Cantu G, Solero CL, Mariani L, et al. (2011) Intestinal type adenocarcinoma of the ethmoid sinus in wood and leather workers: A retrospective study of 153 cases. Head Neck 33: 535-542.

9. Dulgerov P, Jacobsen MS, Allal AS, et al. (2001) Nasal and paranasal sinus carcinoma: Are we making progress? A series of 220 patients and a systematic review. Cancer 92: 3012-3029.

10. Sklar EM, Pizarro JA (2003) Sinonasal intestinal-type adenocarcinoma involvement of the paranasal sinuses. AJNR Am J Neuroradiol 24: 1152-1155.

11. Gras Cabrerizo JR, Orus Dotu C, Montserrat Gili JR, et al. (2006) Epidemiologic analysis of 72 carcinomas of the nasal cavity and paranasal sinuses. Acta Otorrinolaringol Esp 57: 359-363.

12. Fasunla AJ, Lasisi AO (2007) Sinonasal malignancies: A 10-year review in a tertiary health institution. J Natl Med Assoc 99: 1407-1410.

13. Calderon-Garciduenas L, R Delgado, A Calderón-Garcidueñas, et al. (2000) Malignant neoplasms of the nasal cavity and paranasal sinuses: A series of 256 patients in Mexico City and Monterrey. Otolaryngol Head Neck Surg 122: 499-508.

14. Boussen H, A Khedim, S Touati, et al. (2006) Epidemiology of nasal and paranasal sinus cancer in Tunisia. Ann Otolaryngol Chir Cervicofac 123: 115-119.

15. Blanco Al, KS Clifford Chao, Gokhan Ozyigit, et al. (2004) Carcinoma of paranasal sinuses: Long-term outcomes with radiotherapy. Int J Radiat Oncol Biol Phys 59: 51-58.

16. Betlejewski S, Robert Bilewicz, Czesław Stankiewicz, et al. (2006) Malignant tumors of the nose and paranasal sinuses in the years 1992-2001. Otolaryngol Pol 60: 729-736.

17. Lund VJ, Howard D, Wei W (2014) Tumours of the nose, sinuses and nasopharynx. 1-595.

18. Rampinelli V, Ferrari M, Nicolai P (2018) Intestinal-type adenocarcinoma of the sinonasal tract: An update. Curr Op in Otolaryngol Head Neck Surg 26: 115-121.

19. Bossi P, Saba NF, Vermorken JB, et al. (2015) The role of systemic therapy in the management of sinonasal cancer: A critical review. Cancer Treat Rev 41: 836-843.

20. Almeyda R, Capper J (2008) Is surgical debridement and topical 5 fluorouracil the optimum treatment for woodworkers' adenocarcinoma of the ethmoid sinuses? A case-controlled study of a 20-year experience. Clin Otolaryngol 33: 435-441. 